mediastudies.press • Social Media \& the Self: An Open Reader

\title{
Mirror of Your Mind
}

Isabel Munson

Published on: Feb 01, 2021

License: Copyright @ All rights reserved. (COPYRIGHT) 
GAZING INTO TIKTOK'S For You Page-an algorithmically curated, personalized feed that shows videos the platform thinks you will like, learning over time what kind of content will increase your engagement-can be like looking into a hall of mirrors. It's hard to know if its at times uncanny accuracy stems from superior analytics and data harvesting (which are widely mythologized and which companies are often incentivized to oversell) or from the fact that most users can be guided to generally predictable categories. On TikTok, comments like "so we're really all living the same life huh?" are not uncommon. There may also be an availability bias to those occasions when the algorithm guesses really, really right: We remember those, while forgetting the videos that didn't click.

Sometimes the For You Page algorithm is extremely literal, clumsy, and obvious in its techniques: Watch a video of a girl who happens to be a hijabi; be presented with three more hijabis the next day. Despite this, the algorithm can begin to seem to know users better than they know themselves. Its occasional clumsiness and errors in taste may lower users' defenses, creating a randomness that may offset the creepiness of being accurately pigeonholed.

Whatever the reason for its power, the For You Page is central to TikTok's success, which in turn further enhances the oracular aura of its algorithms in a kind of selffulfilling prophecy. What you see on TikTok can then make you question who you are, as if the app must know something you don't. The crystal-ball effect of TikTok can be especially strong when the algorithm suggests something surprising: Users wonder, why does TikTok think I'm ___ (bisexual, a man, a parent of two kids, an alcoholic, ADHD, etc.). Searching Twitter for "TikTok thinks I'm" turns up many examples of people bemused by the algorithm mistaking their identity.

Through its algorithmic recommendations, TikTok essentially diagnoses thousands of people of all ages with medical conditions by showing them, unprompted, videos about ADHD, autism, and more. It's easy to find comments, for example, on an ADHD video along the lines of "I don't think I have ADHD but I keep seeing these videos and it's making me question." The substance of these videos vary wildly, ranging from the surprising ways ADHD and autism may present and how these conditions are underdiagnosed in women, to ways an individual might "mask" their illness, to management tips, like how to organize when you have depression, how to stop a panic attack, or techniques for breaking an overwhelming to-do list down into manageable 
steps. Users may provide a tour of their filthy "depression room" or share what mania looks like for them.

TikTok is rich with such mental health and self-help content, perhaps because the app's direct and intimate format lends itself to such material. Clips often consist of a person's face and torso, close to the camera as though they are FaceTiming a friend, and there is no time or need for fancy intros like on YouTube. There's just someone quickly and eagerly spilling their experiences, presumably in the hope that it helps you. Because videos on the For You Page are strictly served algorithmically, they're often from strangers. In the same way that sharing personal information with a stranger can feel easier than opening up to friends, seeing videos from strangers may allow users to forget what they think they know and just listen to what is being said.

Videos explaining how symptoms of ADHD may present themselves have resonated with hundreds of thousands of women; the comment sections are full of testimonials like "This explains my whole life" or "I just went to the doctor and got diagnosed, I'm 32 and wish I knew this earlier."

I had a similar feeling: Until TikTok's For You Page served me these videos, I didn't knew that ADHD individuals have a different brain chemistry and structure as well as chronically lower dopamine levels, which contribute to distractibility and greater risks of addiction. ADHD symptoms also commonly mirror those of trauma and CPTSD, and women are frequently undiagnosed with ADHD. Basically, I felt like I was hearing all my problems explained to me in under a minute. Years of therapy had never given me such insight.

The experience of TikTok itself may come to feel therapeutic: In some ways it reverses the pressure that platforms like Instagram place on users to show only their best moments, prettiest pictures, and most desirable experiences. On TikTok, your most embarrassing story or childhood photo can become a piece of social capital, a ticket to virality. The platform's proliferation of tremendously weird, "unflattering," and simply "normal" content can feel like a relief. It also cultivates a sense of anonymity, a confessional environment, given that most people don't primarily use it to connect with people they know IRL.

Many mental health professionals have praised the platform for offering accessible information that is destigmatizing and reaches people who otherwise might not be open to therapy or have access. I was served a video of a teenage girl sharing her large bald spot from trichotillomania, a condition that I've had for over a decade. The 
comments section was full of hundreds of people saying that they thought it was just them, saying that they had no idea it was a disease, and thanking the user for bravely sharing her experience. I've also been served videos about people obsessing over their pores and popping pimples in the mirror, a condition called dermotillomania. Again, the comments section was packed with young people who didn't know this was a recognized disorder. Understanding that "it's not just me" or "not my fault for being like this" can be a tremendous relief.

At the same time, receiving mental health advice from TikTok can be problematic. The videos are one minute at most, designed for virality, and not vetted by a therapy board or peer review. Anyone can make a mental health video, and the simplistic, short-form nature of the content risks implying that the experience shared within is universal or that the individual is an expert. In addition, the algorithm is expressly designed to present people with information they want to hear. It is not likely to present challenging rebuttals or prompt questioning of their stance. In therapy, your therapist may push back against what you say. On a platform designed to increase engagement and keep people hooked, that seems unlikely.

TikTok is a space designed to create confirmation bias-or more accurately, engagement bias. Whereas a therapist might question the usefulness of identifying oneself as permanently aligned with whatever struggle one is experiencing, engagement-driven platforms help frame conditions as points of identity, badges of honor. If users find they are rewarded primarily for producing content on a certain condition, belief, or identity, it can skew their motivations and self-definition, making them believe at some level that it is the most interesting and likable thing about them. Some individuals exaggerate or fake "tics" or "stimming" for the likes, or they indulge in self-diagnosing with certainty. As much as it's important to destigmatize neurodivergence and mental illness, there are risks when these struggles are glorified for clout or presented as static and unchangeable. There's a fine line between destigmatizing and incentivizing, especially on social media platforms with built-in feedback mechanisms.

The diagnostic procedures of TikTok are not limited to showing some users videos that pertain to specific medical conditions. The logic of diagnosis is embedded in every piece of content, each new piece of content functioning as a probe to reveal and assign personality traits, potential hobbies, and obsessions. Identity itself is configured as a condition to be uncovered rather than articulated or expressed. 
With how routinely social media platforms are now derided for fomenting surveillance, conspiracy, and polarization, it can be easy to forget that they were once heralded for ushering in a new emphasis on self-expression. If you signed up you could "share" your activities and ideas to a wider range of people and seem to expand the contours of what the self could be. Initially, this process was a novelty, but as social media became more routine, skillful self-presentation became a familiar and necessary skill, almost a self-care ritual, driven by how other people and algorithms respond to your posts.

With traditional markers of life progress and self-definition-home ownership, marriage, kids, stable careers-becoming less normative as well as less broadly attainable, it can make sense to treat social media as an opportunity to restabilize identity, a chance to turn inward toward something we can control: the aesthetic and the personality we project, the labels we apply to ourselves. Many social media bios are a collection of such identifiers: astrology sign, pronouns, gender and sexual orientation, political orientations, mental illnesses, disabilities, location, age, MyersBriggs type, fandoms, jobs, hobbies, and more.

That is to say, social media platforms drive not just self-expression but self-discovery. They are a means of production for identity, which affects our sense of what identity is for and what motivates how we refine it. For example, user profiles are both uniquely personal yet also deeply standardized, and the density of social connection prompts both a demand to post creatively and to conform to popular formulas for content. This drives the pursuit of uniqueness and niche identities, as self-differentiation represents a profit opportunity, much like product differentiation within advertising.

Just as capitalism engenders the belief that our value is determined by our productivity, "social" as a business category influences our concept of the self, encouraging us to see self-categorization on platforms not only as self-realization but as a source of capital. Our value then is based on effective self-branding, on the ongoing ability to reproduce the stable and refined self we project online. Mastery of this process has itself become a career option: "influencing," which requires a dedication to producing content and a talent for translating a persona into legible posts. The more easily understood the persona, the wider an audience it can reach.

The capitalist logic of platforms and their use as a means of self-discovery are thus deeply interconnected. Just as the apps seek to categorize users, personalize content, and accurately target them with advertising, users seek to categorize their self, interests and identities and accurately convey their essence in a profile. This model of the self mimics that of the feed-an endless source of content that will keep flowing, 
generating data, refining, indexing, and articulating identity. The platform's business tools-like recommended content and people to follow-are presented as helpful tips for further discovery and refinement. Algorithms and engagement metrics act as feedback mechanisms, aiding the user in articulating their self-categorization by perpetually refining new content and evaluating its effectiveness.

No platform is more committed to this-or successful at it-than TikTok. On Twitter and Instagram, the user self-selects what and who they want to follow and how they want to define themselves. Though feeds are sorted algorithmically and the platforms recommend content and topics, users' feeds are primarily propagated with accounts they've chosen to follow. On TikTok, however, the For You Page algorithm does all the categorizing for you, steering you down paths it thinks will lead to the most engagement.

Eugene Wei ascribes TikTok's success to this distinction, and the app's unprecedented ability to surface content from users' "interest graph" rather than their "social graph," like other platforms have. By creating an experience based chiefly on catering to user's interests, with zero on-boarding required other than scrolling, TikTok avoided common friction points in creating a popular network, like the potentially anxiety-inducing challenge of personally curating your feed, given what such choices say about your economic value.

While the majority of attention on the app goes to the mainstream "straight TikTok," "full of hype houses, viral dances, and accounts with millions of followers, like Charli D'Amelio and Chase Hudson ("Lil Huddy")-TikTok also generates countless niche culture-less subcultures, sometimes from a single video. FrogTok comes to mind, but there's also “cottagecore” TikTok, Prison TikTok, WitchTok, Anti-Lawn TikTok, Bean TikTok, DeepTok, Foraging TikTok, CartelTok, to name a few. Recently, Sea Shanty TikTok experienced a wave of popularity.

People have even made videos mapping out the journey the algorithm has taken them through different TikTok micro-communities. These maps define a landscape of different content destinations that are passed through or avoided on the quest to reach the "deepest" levels of TikTok, with the weirdest, rarest, or "best" content. Searching the phrase "You've reached" on TikTok reveals a huge variety of these declarations, with creators or commenters defining a topic or genre as a destination. These different TikTok subcultures are typically trends that don't last as a genre, but the urge to label what "side" of TikTok a user has stumbled upon reveals the desire to define stable categories and destinations amid the flux, to aid users with future articulation of their 
own tastes and identity. The fixation on reaching the deepest sides of the platform reflects the emerging fantasy of reaching the source of a stream that is endless by design.

Identity, too, is accordingly structured as endless and unstable; one never reaches the final destination where they have "completed" evolving. The lack of a finish line is not a deterrent; users instead continually try to turn endlessness into stability. The constantly refining feed becomes a stable backdrop, and the quest for a "solved" fixed identity becomes perpetual.

It's paradoxical to seek stability within platforms that incentivize flux, virality, and mimetic copying. But TikTok, and algorithmic feeds in general, make this broader contradiction seem coherent: Identity can be both stable (who you "really" are) and constantly changing (a form of "human capital" that can be accumulated and manipulated as circumstances demand).

There is something sinister about the algorithmically supported push to define, diagnose, declare, and "solve" the self. Medical diagnosis and definition can absolutely be valuable, even life-saving. But when everything is indexed and flattened to a profile, we enter the passive role of content consumer surfing the waves of infinite new videos and posts to try and find ourselves within.

The search for the self is now framed as a pastime, easy to pursue, and embedded within the logic of the platforms down to the design of interfaces, algorithms, and features. But this search runs counter to the idea that the best way to find value in oneself is by becoming a part of the whole-connecting with others, helping others, and shedding the idea of a "self" distinct from the rest of the world. In theory, platforms could cultivate this sense of interconnectedness and intrinsic worth in every individual, as people are more ubiquitously connected and networked than ever, but platforms instead tend to promote differences, create niches, and elevate conflict and division for engagement.

Without ever arriving at this insight, people may be resigned to constantly solving and diagnosing themselves, condemning others, and getting lost in the slipstream of online spaces in an attempt to make sense in a senseless world. Knowledge is power, but an endless stream of videos and tips and tricks can end up serving as compulsive distraction. You pry yourself away from the feed as if from a fugue state, both having learned everything and nothing. 
Isabel Munson is a multimedia artist based in New York City. She writes and makes documentaries examining relationships between technology, design, economics and behavior. She also produces music, DJs, and runs NYC-based record label Worst Behavior Recs.

\section{REPRINT}

"Mirror of Your Mind" (Isabel Munson, Real Life, February 1, 2021)

REPRINTED WITH PERMISSION FROM REAL LIFE, WHICH RESERVES ALL RIGHTS 